\title{
IMAGING
}

\section{Warming up to a glow}

New dyes emit near-infrared chemiluminescence when warmed to body temperature, allowing deep-tissue imaging in mice.

Fluorescence imaging deep within a living animal is not easy. The problem with light is that it does not penetrate very far through tissue; at depths beyond a few millimeters below the surface, scattering of the excitation light obscures the signal. The alternative for deep imaging, the use of radiotracers, comes with serious safety concerns.

Bradley Smith, his student Jeffrey Baumes, and their colleagues at the University of Notre Dame now report a new class of nearinfrared, chemiluminescent dyes that allow deep-tissue imaging. These dyes are based on a molecular architecture known as a rotaxane, consisting of a dumbbell-shaped squaraine dye, encapsulated by a "bulletproof vest" (as Smith puts it) of a macrocycle. Smith and Baumes were attempting to make red and near-infrared dyes for fluorescence imaging in animals when they discovered that these molecules had unique properties. When exposed to light, the dye reacts with oxygen to produce an endoperoxide, which in turn releases singlet oxygen and emits near-infrared chemiluminescence. However, the endoperoxide is stable below $-20^{\circ} \mathrm{C}$ such that the dye can be generated in a test tube and then stored. When injected into an animal, the dye warms up and generates the signal without the need for any excitation light.

The team packed the squaraine rotaxane endoperoxide dyes inside microparticles and tested their application for imaging in living mice. "What we discovered with the system is that the light will penetrate much, much further, all the way through the animal," explains Smith. "It goes through bones, organs; a lot of things that typically scatter light or absorb it."

Another beneficial property of these dyes is they also remain highly fluorescent, notes Smith: "You can use them in chemiluminescence mode for deep-tissue imaging and then switch over to the fluorescence mode for shallow imaging." The researchers also have preliminary evidence that the chemiluminescence can be regenerated, a property that could be exploited to enhance detection sensitivity.

Smith is interested in exploring many applications of these dyes. He hopes to be able to image deep-tissue tumors in mice, for example, and foresees potential clinical applications of the technology down the road. In the nearer future, he believes that the enhanced sensitivity of these chemiluminescent dyes will find a variety of in vitro uses in the lab.

\section{Allison Doerr}

\section{RESEARCH PAPERS}

Baumes, J.M. et al. Storable, thermally activated, near-infrared chemiluminescent dyes and dyestained microparticles for optical imaging. Nat. Chem. advance online publication 24 0ctober 2010. 